\title{
Variazioni epidemiologiche delle neoplasie tiroidee nel tempo: ruolo della nutrizione iodica
}

\author{
Giorgio Grani ${ }^{1} \cdot$ Valeria Ramundo $^{1} \cdot$ Rosa Falcone $^{1} \cdot$ Cosimo Durante $^{1}$
}

Accettato: 4 settembre 2020 / Pubblicato online: 24 febbraio 2021

(c) The Author(s) 2021

\section{Introduzione}

Lo iodio è cruciale per l'omeostasi tiroidea: oltre ai noti effetti sulla funzione, la deficienza cronica di iodio può favorire lo sviluppo di gozzo o un aumento di prevalenza ed incidenza di noduli tiroidei benigni [1]. La relazione con il carcinoma tiroideo è più controversa e gli studi che hanno indagato questa associazione sono discordi: una revisione della letteratura sull'argomento è stata recentemente pubblicata [2]. Alcuni studi pubblicati negli anni '90 avevano documentato un rischio incrementato di carcinoma tiroideo nelle popolazioni residenti in aree iodocarenti; in particolare, la distribuzione dei vari istotipi risultava sbilanciata verso una maggiore prevalenza di carcinoma follicolare. Inoltre, alcuni studi longitudinali, effettuati prima e dopo l'introduzione di programmi di iodoprofilassi, hanno documentato un incremento relativo dei carcinomi a rischio più basso (in particolare i carcinomi papilliferi della tiroide). I risultati riportati in letteratura sono comunque discordanti. L'interpretazione di eventi che avvengono durante ampi intervalli temporali, infatti, risulta complicata da molteplici fattori confondenti. Oltre all'implementazione di programmi di profilassi iodica negli anni Ottanta e Novanta, altri fattori possono spiegare la maggior prevalenza (relativa) di carcinomi papilliferi della tiroide. Tali fattori vengono discussi nelle sezioni seguenti e rappresentati in Figura 1 in parallelo all'implementazione dei programmi di profilassi iodica.

$\varangle$ C. Durante

cosimo.durante@uniroma1.it

1 Dipartimento di Medicina Traslazionale e di Precisione, Sapienza Università di Roma, Roma, Italia

\section{Introduzione dell'ecografia del collo ed eccesso di diagnosi}

L'introduzione dell'uso routinario dell'ecografia del collo nella pratica endocrinologica (anni Ottanta) e l'aumentato utilizzo dell'agoaspirato tiroideo ecoguidato hanno portato ad un incremento delle diagnosi di carcinoma tiroideo e, soprattutto, dei microcarcinomi papilliferi, molti dei quali sarebbero stati clinicamente silenti in assenza dello screening ecografico. L'incremento a livello mondiale nell'incidenza del carcinoma papillifero della tiroide è stato documentato nei paesi con apporto iodico stabile, crescente o in riduzione [3]. Uno studio basato sul registro svedese ha mostrato che i programmi di supplementazione iodica (in quel paese intrapresi già nel 1936 e ulteriormente rafforzati nel 1966) hanno prodotto un appiattimento delle differenze regionali, eliminando l'eccesso di carcinomi follicolari presenti nelle aree a maggior iodocarenza (il rischio relativo era pari a 1.98 negli uomini e 1.17 nelle donne). Tuttavia, l'incidenza di entrambi gli istotipi papillare e follicolare è aumentata fra il 1958 e il 1981, con tendenze simili nelle aree iodo-sufficienti e iodocarenti [4].

\section{Cambiamento delle definizioni anatomo-patologiche e definizione della variante follicolare del carcinoma papillifero}

Fino agli anni Settanta, la definizione di carcinoma follicolare e papillifero era basata sul solo pattern di crescita. Solo nel 1977, Chen e Rosai hanno introdotto la variante follicolare del carcinoma papillifero, rendendo la morfologia nucleare un criterio per la classificazione istologica, indipendentemente dalla prevalenza degli aspetti di crescita follicolare. A seguito di questa variazione, molti carcinomi 


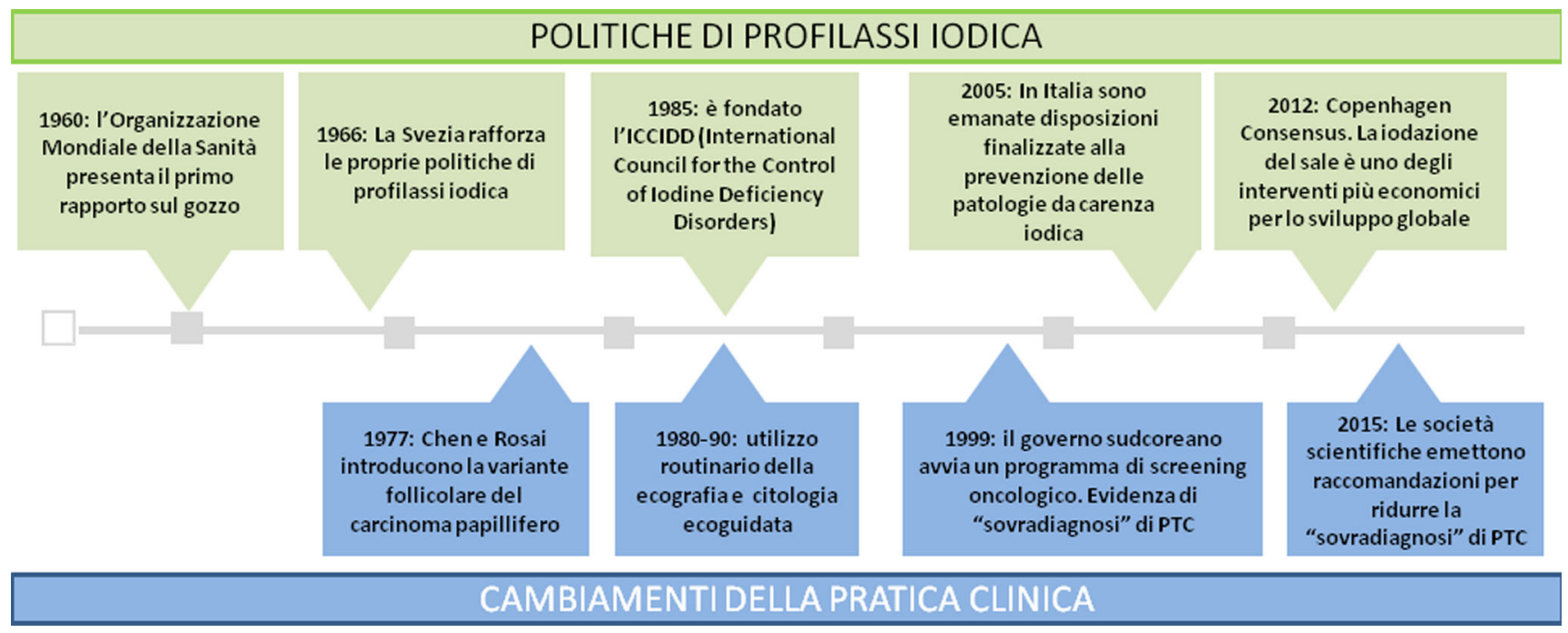

Fig. 1 Politiche di profilassi iodica e cambiamenti nella pratica clinica verificatisi parallelamente nel corso degli ultimi decenni

precedentemente classificati come follicolari, sarebbero oggi classificati come papilliferi. Uno studio di Verkooijen e colleghi [5] ha dimostrato che fino al $45 \%$ dei carcinomi follicolari diagnosticati negli anni $70-80$, sarebbero riclassificati come papilliferi a seguito di revisione alla luce delle classificazioni successive.

\section{Conclusioni}

Il cambiamento del rapporto follicolari/papilliferi a favore di questi ultimi risente dell'effetto combinato dell'incremento delle diagnosi di microcarcinoma papillifero "subclinico", dei cambiamenti dei criteri anatomopatologici [6] oltre che della differente supplementazione iodica, con una rilevanza relativa che è difficile da determinare utilizzando i dati attualmente a disposizione.

La valutazione dell'apporto iodico è complessa: idealmente da valutare attraverso la misura dell'escrezione urinaria di iodio nella popolazione di riferimento, è nella maggior parte degli studi stimata indirettamente tramite l'utilizzo di questionari nutrizionali, notoriamente proni al recall bias, o basata sulla semplice frequenza del consumo di prodotti ittici come marcatore surrogato. Negli studi in cui invece l'apporto iodico viene calcolato sulla base dei dati di popolazione di specifiche regioni, l'impatto di altri fattori (geografici, come la presenza di aree vulcaniche o di attività industriali; sociali, come il livello di urbanizzazione e socioculturale medio; la disponibilità e l'efficienza dei sistemi sanitari) è difficile da determinare e, quindi, da considerare nelle analisi statistiche.

Le politiche che mirano a promuovere la nutrizione iodica hanno dimostrato, in uno studio europeo, di aumentare l'aspettativa di vita (corretta per la qualità di vita) nelle aree iodo-carenti, a livello di popolazione [7]. Esse possono ridurre l'incidenza e la prevalenza del gozzo (e forse alcune forme di patologia nodulare tiroidea), ma non sembrano esercitare un ruolo significativo nella riduzione del rischio di carcinoma tiroideo. Altri fattori (sia genetici che ambientali) hanno una maggiore rilevanza nello spiegare il cambiamento del profilo dei carcinomi tiroidei.

Funding Note Open access funding provided by Università degli Studi di Roma La Sapienza within the CRUI-CARE Agreement.

Conflitto di interesse Gli autori dichiarano di non avere conflitti di interesse.

Consenso informato Lo studio presentato in questo articolo non ha richiesto sperimentazione umana.

Studi sugli animali Gli autori di questo articolo non hanno eseguito studi sugli animali.

Nota della casa editrice Springer Nature rimane neutrale in riguardo alle rivendicazioni giurisdizionali nelle mappe pubblicate e nelle affiliazioni istituzionali.

Open Access This article is licensed under a Creative Commons Attribution 4.0 International License, which permits use, sharing, adaptation, distribution and reproduction in any medium or format, as long as you give appropriate credit to the original author(s) and the source, provide a link to the Creative Commons licence, and indicate if changes were made. The images or other third party material in this article are included in the article's Creative Commons licence, unless indicated otherwise in a credit line to the material. If material is not included in the article's Creative Commons licence and your intended use is not permitted by statutory regulation or exceeds the permitted use, you will need to obtain permission directly from the copyright holder. To view a copy of this licence, visit http://creativecommons.org/licenses/by/4.0/. 


\section{Bibliografia}

1. Laurberg P, Cerqueira C, Ovesen L, Rasmussen LB, Perrild H, Andersen $S$ et al (2010) Iodine intake as a determinant of thyroid disorders in populations. Best Pract Res Clin Endocrinol Metab 24(1):13-27

2. Barrea L, Gallo M, Ruggeri RM, Giacinto PD, Sesti F, Prinzi $\mathrm{N}$ et al (2020) Nutritional status and follicular-derived thyroid cancer: an update. Crit Rev Food Sci Nutr 61(1):25-59. https://doi.org/10.1080/10408398.2020.1714542

3. Zimmermann MB, Galetti V (2015) Iodine intake as a risk factor for thyroid cancer: a comprehensive review of animal and human studies. Thyroid Res 8:8

4. Pettersson B, Coleman MP, Ron E, Adami HO (1996) Iodine supplementation in Sweden and regional trends in thyroid cancer incidence by histopathologic type. Int J Cancer 65(1):13-19
5. Verkooijen HM, Fioretta G, Pache JC, Franceschi S, Raymond L, Schubert $\mathrm{H}$ et al (2003) Diagnostic changes as a reason for the increase in papillary thyroid cancer incidence in Geneva, Switzerland. Cancer Causes Control 14(1):13-17

6. Grani G, Lamartina L, Durante C, Filetti S, Cooper DS (2018) Follicular thyroid cancer and Hürthle cell carcinoma: challenges in diagnosis, treatment, and clinical management. Lancet Diabetes Endocrinol 6(6):500-514

7. Schaffner M, Mühlberger N, Conrads-Frank A, Qerimi Rushaj V, Sroczynski G, Koukkou E et al (2020) Benefits and harms of a prevention program for iodine deficiency disorders - predictions of the decision-analytic EUthyroid model. Thyroid. https://doi.org/ 10.1089/thy.2020.0062 